\title{
Identification of Magnetic Dipole Innes Above 2000 A in Several Mo A.Id zr Ions on the PLT Tokamak
}

S. Suckewer, E. HInnov, S. Cohen,

M. Finkenthal ", and R. Sato"*

PPPL- -1899

DE82 014865

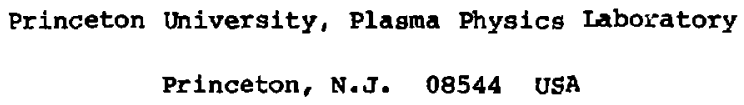

Abstract

\begin{abstract}
A number of spectrum lines arising from magnetic dipole transitions in the $\mathrm{n}=3$ shell ground conficurations of molybdenum and zirconium Ions have been identified. These lines are particulariy suitable for spectroscopic diagnostics in tokamak-type plasnas in the 500-1500 eV temperature range.
\end{abstract}

\footnotetext{
"Visitor from Racah Institute of Physica, The Hebrew University, Jerusajem, Israel.

visitor from Nagoya University, Institute for Plagra Fhysics, Japan.
}

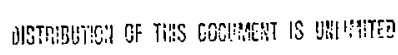


The relatively long wavelength magnetic dipole lines of the $n=2$ sheil of highly tonizec elements from scandiun to copper $(z=21-29)$ are being extensively used for local1zed plasma diagnostics in tokamaks. 1-3 in this respect, lines with wavelengths above the air cutoff, $\lambda>2000 \AA$, offer special advantages because of the relative simplicity and versatility of the applicable instrumentation. However, in the new generation tokama s, with expected peak temperatures above $5 \mathrm{keV}$, the $\mathrm{n}=2$ shells of these eleme.its are stripped ove the most interesting part of the plasma, thus necessi ating diagnostic use of sonewhat heavier elements, perhaps up to molybdenum $(z=42)$. In such heavier elements, the magnetic dipole transitions in the $n=3$ shell are also sufficiently intense for spectroscopic measurements, tus providing the means of simultaneous diagnostics of the outer lower-temperatc-e region of the plasma. Also, in the presently operating tokamaks, witn $T_{e}(0) \sim 1-3 \mathrm{keV}$, it may be of interest to compare measurements in the same location with two elements of slgnificantly different mass, e.g., the $n=3$ states of $N_{0}$ or $\mathrm{Zr}$ and the $n=2$ states of $\mathrm{TI}$ or $\mathrm{Sc}$.

In the present paper we report the ldentiflcation of a number of $n=3$ shell magnetic dipole lines, with wavelengths above the air cutoff, of varlous Mo and Zr ions, whlch were introduced into the PLT tokamak alscharges for dlagnost1c purposes.

Figure 1 shows the mear ared wavelengths (1n boxes) and the trangitlons in the molybdenum lons, which we consider falrly reliably 1dentified. The saga of discovery of these 11 nes $1 \mathrm{~s}$ too varied to be recounted here, but $1 t$ consists of a combination of 1soelectronic extrapolations (which axe more vague and unreliable than in the relatively simple $n=2$ sheli) from available data in lower-Z elements, ${ }^{4-7}$ measurement of time-erolution and radial profiles of the entssivities of these IInes, some of which will be presented below, and 
finally the internal consistercy of the corresponaing transitions in molybdenum and zircontum ions. One exception to this process was the $3 p^{6} 3 d^{8}$ $3_{\mathrm{F}_{3}}+3_{\mathrm{F}_{4}}$ transition, which had been established from the shorter wavelength measurements of Reader and Ryabtsev, ${ }^{B}$ with which our recults are in excellent agreement.

The experimental. procedure is qualitatively gimllar to the $n=2$ sheil measurements. 9 The element in question is introduced by the laser blowoff method at a preselected time during the discharge, and, as a consequence of the relatively slow radial transport, the 1ncreasing lonization stages appear successively in time, and in quasi-cylindrical shells in radial distribution, determined primarlly by the ionization potentials of the lons (and hence their collisional Ionlzation rates). Helpful boundg for the time and space varlations are provided by the well-establishes resonance lines of the magneslum or sodium sequences on one side and the zinc or copper sequences on the other side, for the ions in question.

A sample of the time behavior of some of these lines $1 \mathrm{~s}$ shown in F1g. 2, with the sodium-like Mo XXXII resonance line given for comparison. (The $\sim 4$ msec perlod modulation on the wo XxIV 2686 A line is caused by a vibrating IIF plate, which sweeps the line 1mage repeatedly across the exit slit. This allows determination of the Doppler width and hence ion temperature at a radial location established from spatial emissivity scan described below.) The time sequence, for both appearance and disappearance, clearly occurs in the ascribed order, although in adjacent states, e.g., MoXXIII and XXIV, the differences are quite small. Thus the time-behavior alone is often not aufficlent for precise assignment of an unknown line, especially a weak one, to a particular lonization state although the range of uncertainty is rather limited. 
Figure 3 shows the radial distribution of the measured emissivities of the three Mo Ion 11neg, together with that of 2271 A line of $C V\left(E_{1}=392 \mathrm{eV}\right)$ for comparison, and the eleatron temperature profile from single-shot Themson scattering. The emisgivity profiles are determined by Abel-inverting chord brightnesses measured by means of a rotating mirror, which scans the plasma vertically with a period of about $10 \mathrm{msec}$. The emissivities clearly occur in fairly distinct radial shells, which again is helpful for the assignment of the ionization atagea for the observed lines, and forms the basis for their use of radially localized diagnostics. The location and shape of thes: shells is nearly constant in time, provided the electron temperature prof 1 le remains unchanged. The peaks occur at $T_{e}(r)$, which $1 \mathrm{~s}$ slightly lower than the Ionization potentials, but distinctly higher than what would correspond to coronal ionization equilibrium. There is evidence of some polotdal or up-down asymmetry both in the emissivities and the electron temperature. The significance of this has not been determined, although it is not primarily due to measurement uncertainties.

We also note that in a discharge with central $T_{e}(0)=1$ keV, the Mo XXIII line emissivity was peaked on the axis, with a radial width of she profile about $\pm 10 \mathrm{~cm}$.

The temperature profile on Fig. 3 is an average of four discharges. Both the $T_{e}(r)$ profile and the so injection had some small but significant shot-to-shot variation. It is therefore not worthwhile to atternpt too quantitative interpretation of these data, e.g., to derfve deviations from coronal equilibriun.

The measured wavelengths (in air) together with egtimated uncertainties are glven in Table I. For tokamak plasma diagnostic purposes, both wo XXIV 2686 \& line and wo XXIII 3553 \& line are relatively strong, and free of 


\begin{abstract}
significant interfering lines. They are thus eminently autable for diagnostics of $\sim 1 \mathrm{keV}$ plasina. Their (2686:3554) measured intensity ratio 1s about $2: 1$, which, in view of the similarity of their radial distribution, is also their enissivity ratio. The 2841 \& Iine of Mo XXIX is also well separated from interfering lines, and it has typically about one-half the Intensity of the 3553 \& 1ine. qualitatively similar statements apply to the corresponding zirconium lines, except that the 3100 \& lne suffers some interference from a thus far unidentifled line in the discharge. We want to emphasize here that the emissivitles are only partly determined by radiative transition rates, i.e., collisional rates, including cascading, have important, indeed usually dominant, effect. Their quantitative Interpretation, especially in the more complicated $d^{2}$ and $d^{8}$ configurations, is very approximate at present.
\end{abstract}

Table I. Obgerved Nolybdenum and (Sircontum) Wavelengths (in $\AA$ ) For Transitions Given By Figure 1.

\begin{tabular}{|c|c|c|c|c|c|}
\hline Mo XVI & Mo XVII & Mo & XXIII & Mo XXIV & Mo $X X I X$ \\
\hline (Zr XIV) & $(\mathrm{Zr} \mathrm{XV})$ & ( $\mathbf{Z r}$ & $X X(1)$ & (Zr XXII) & (Zr XXVII) \\
\hline $3708.1 \pm 0.2$ & $4123.5 \pm 0.3$ & $3553.3 \pm 0.3$ & $3319.8 \pm 0.3$ & $2686.5 \pm 0.3$ & $2841.1 \pm 0.2$ \\
\hline $4967 \cdot 4 \pm 0.3)$ & $(5547.6 \pm 0.3)$ & $(4774.2 \pm 0.4)$ & & $(3507.1 \pm 0.2)$ & $(3100.2 \pm 0.4$ \\
\hline
\end{tabular}

The assignment of the 3320 A line must be regarded as more tentative than the others. It is about 4-5 times weaker than the 3553 \& I1ne. This seems somewhat too weak, although varlous cascading transitions would tend to enhance rather strongly tr: ${ }^{3} F_{3}-{ }^{3} F_{2}$ transition. Furthermore, the corresponding zircontum line wirich would occur at -4431 A if the assignment is 
correct has not yet been observed. The assignment is based on the approximately expected wavelength and the proper time dependence of the emissivity. It was too weak for reliable measurement of intensity radial distribution. In view of the small state-to-state variations of the time dependence it may possibly belong to Mo XXII or even XXI, In which case the $3 d^{2}{ }^{3} F_{4}-{ }^{3} F_{3}$ transition is yet to be discovered.

Acknowledgments

The authors wish to thank $J$. Hosea for supporting these measurements, F. Boody, C. Daughney, D. Dimock, and D. McNell for providing Thomson scattering $T_{e}$ data, the ppr surface physics group for enthusiastic cooperation, and the PLT operators for their acquiescence in the course of these experiments.

This work was supported by the Department of Energy, Contract No. AC02-76-CHO-3073. 
References

1s. Suckewer, Physica Scripta 23, 72 (1981).

2E. Hinnov, R. Fonck, s. Suckewer, Bull. Am. Phy Princeton Flasma Physics Laboratory Report 1669 (

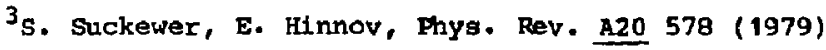

${ }^{4}$ J. Reader and J. Sugar, J. Phyg. Chem. Ref. Data

${ }^{5}$. Sugar and C. Corliss, J. Phys. Chem. Ref. (1981).

${ }^{6}$ J.R. Fuhr, G.A. Martin, W.L. Wiese, S.M. Younger, $305(1981)$.

7R. Smitt, L.A. Svensson, M. Outred, Physica Scr1p

8. Reader and A.N. Ryabtsev, J. Opt. Soc. Am. 71

9. Hinnov, S. Suckewer, S. Cohen, K. Sato, Phys press). 


\section{Flgure Captions}

Fig. 1 Measured wavelengths (In A) and transitions ascribed to several molybdenum lons. $E_{1}$ are the approximate lonization potentials of these Ions. The wavelengths in parentheses for Mo xxIX are predicted values.

Fig. 2 Observed time behavior of several molybdenum lon lines after the injection. The rapid modulation of the Mo XXIV is described in the text. The small osclilations, e.g., on to XXIX line 10-30 msec are probably cauged by radially localized MHD plasma oscillations.

Fig. 3 Local emissivities of tho XVII, XXIII and XXIX ions and CV $2271 \AA$ 1Ine, w1th the radial electron temperature profile from Thomson scattering. The electron density is about $2 \times 10^{13} \mathrm{~cm}^{-3}$ near the center and slightly sharper than parabolic in shape within the \pm 40 on limiter radius. 


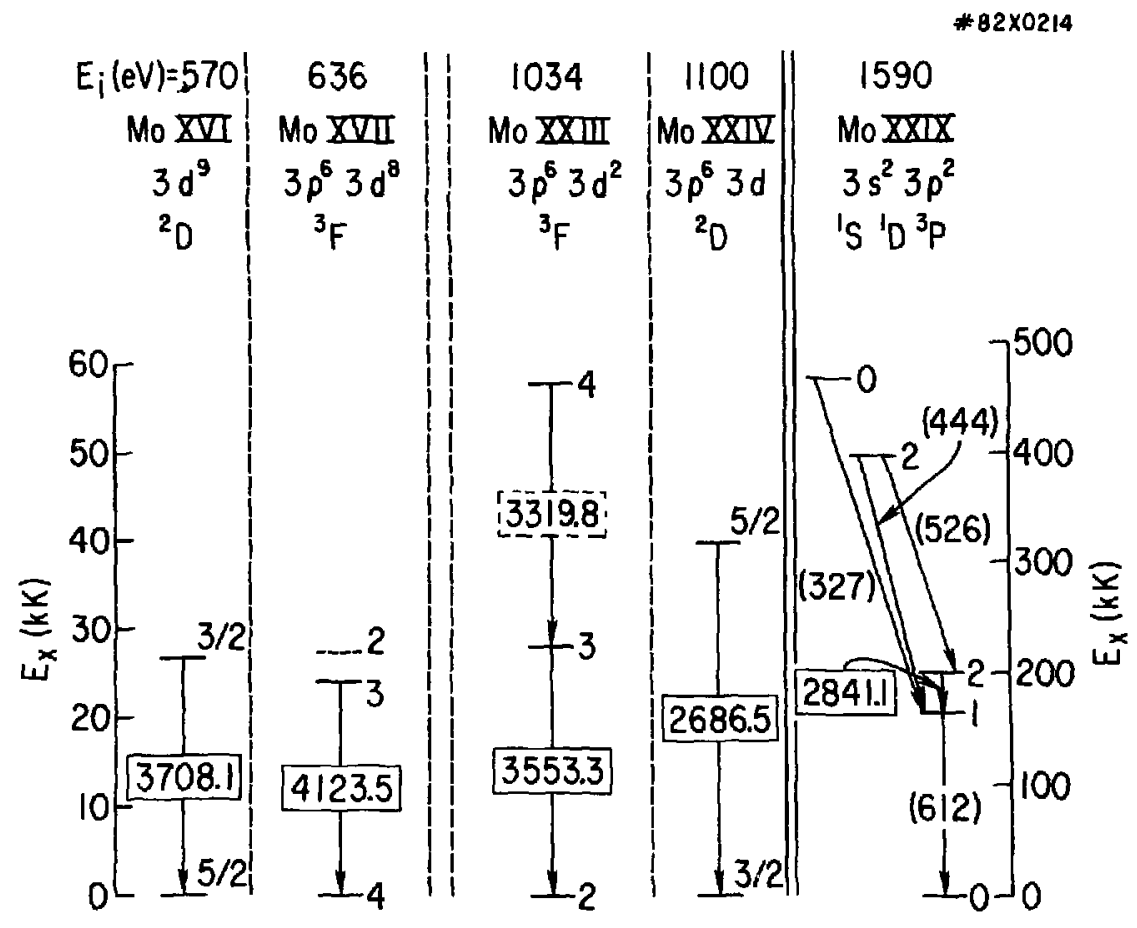

Fig. 1 


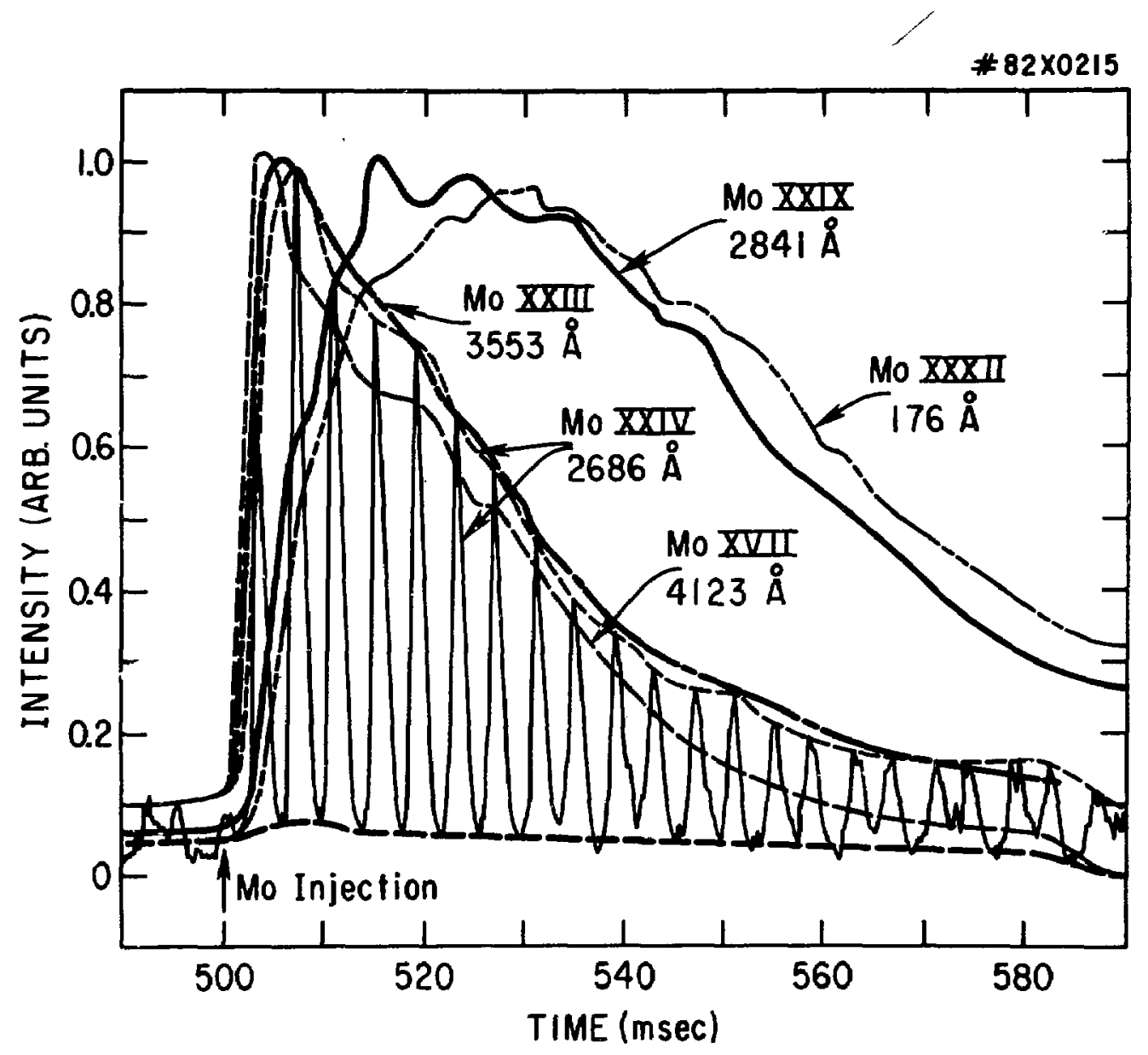

Fig. 2 
\# $82 \times 0216$

PLT, FAST RADIAL SCAN (ABEL INVERTED)

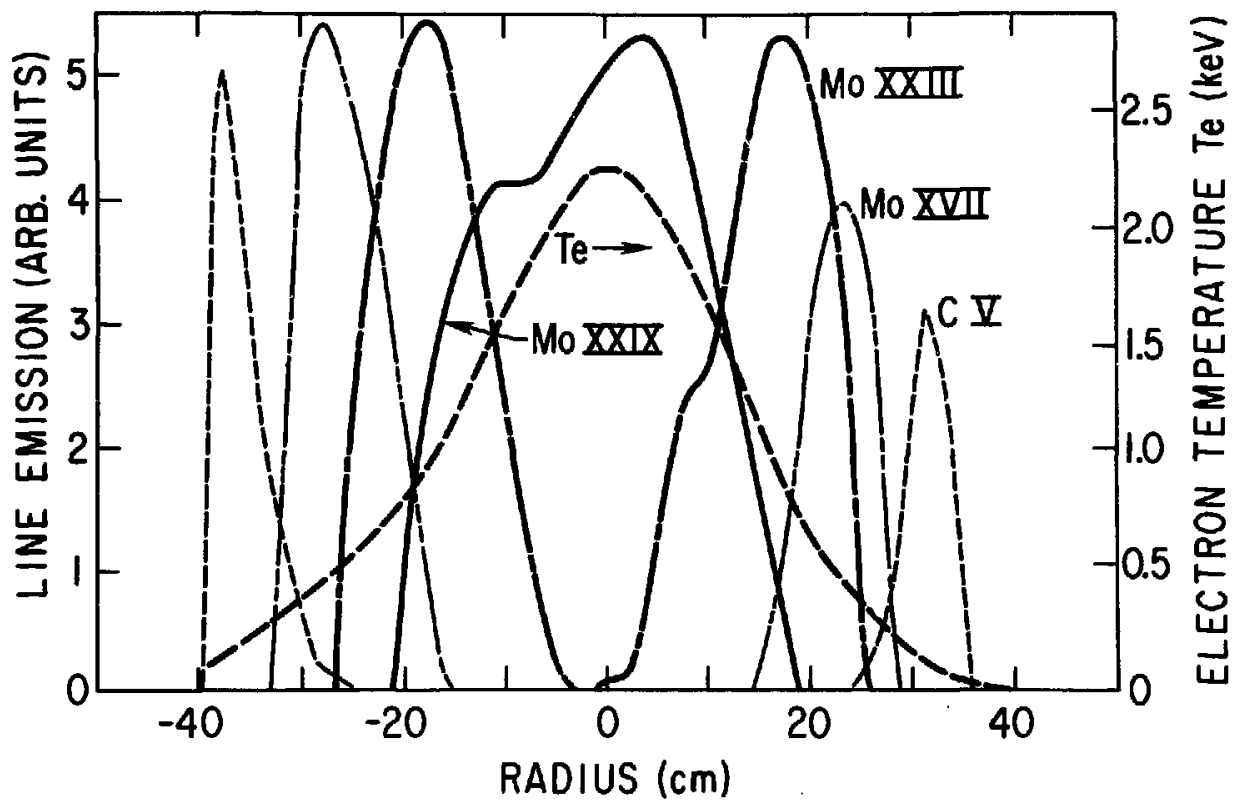

Fig. 3 